\title{
Erratum to: ASNC imaging guidelines for SPECT nuclear cardiology procedures: Stress, protocols, and tracers
}

\author{
Milena J. Henzlova, $M D,{ }^{a} W$. Lane Duvall, $M D,{ }^{b}$ Andrew J. Einstein, $M D,{ }^{c}$ \\ Mark I. Travin, $M D{ }^{d}$ and Hein J. Verberne, $M D^{\mathrm{e}}$ \\ a Mount Sinai Medical Center, New York, NY \\ b Hartford Hospital, Hartford, CT \\ c New York Presbyterian Hospital, Columbia University Medical Center, New York, NY \\ ' Montefiore Medical Center, Albert Einstein College of Medicine, Bronx, NY \\ e Academic Medical Center, Amsterdam, The Netherlands
}

doi: $10.1007 / \mathrm{s} 12350-016-0463-x$

\section{ERRATUM TO: J NUCL CARDIOL DOI 10.1007/S12350-015-0387-X}

Please note the correction in Table 5 of the published article. Under the heading "Second Injection', and subhead "Activity $(\mathrm{MBq})$,' the third row of numbers, which currently reads "'888-1332," should read ' $296-444$ ', The correct Table 5 is given here.

The online version of the original article can be found under doi:10.1007/s12350-015-0387-x.

Reprint requests: Milena J. Henzlova, MD, Mount Sinai Medical

Center, New York, NY; Milena.Henzlova@mssm.edu

J Nucl Cardiol 2016;23:640-2.

$1071-3581 / \$ 34.00$

Copyright (C) 2016 American Society of Nuclear Cardiology. 


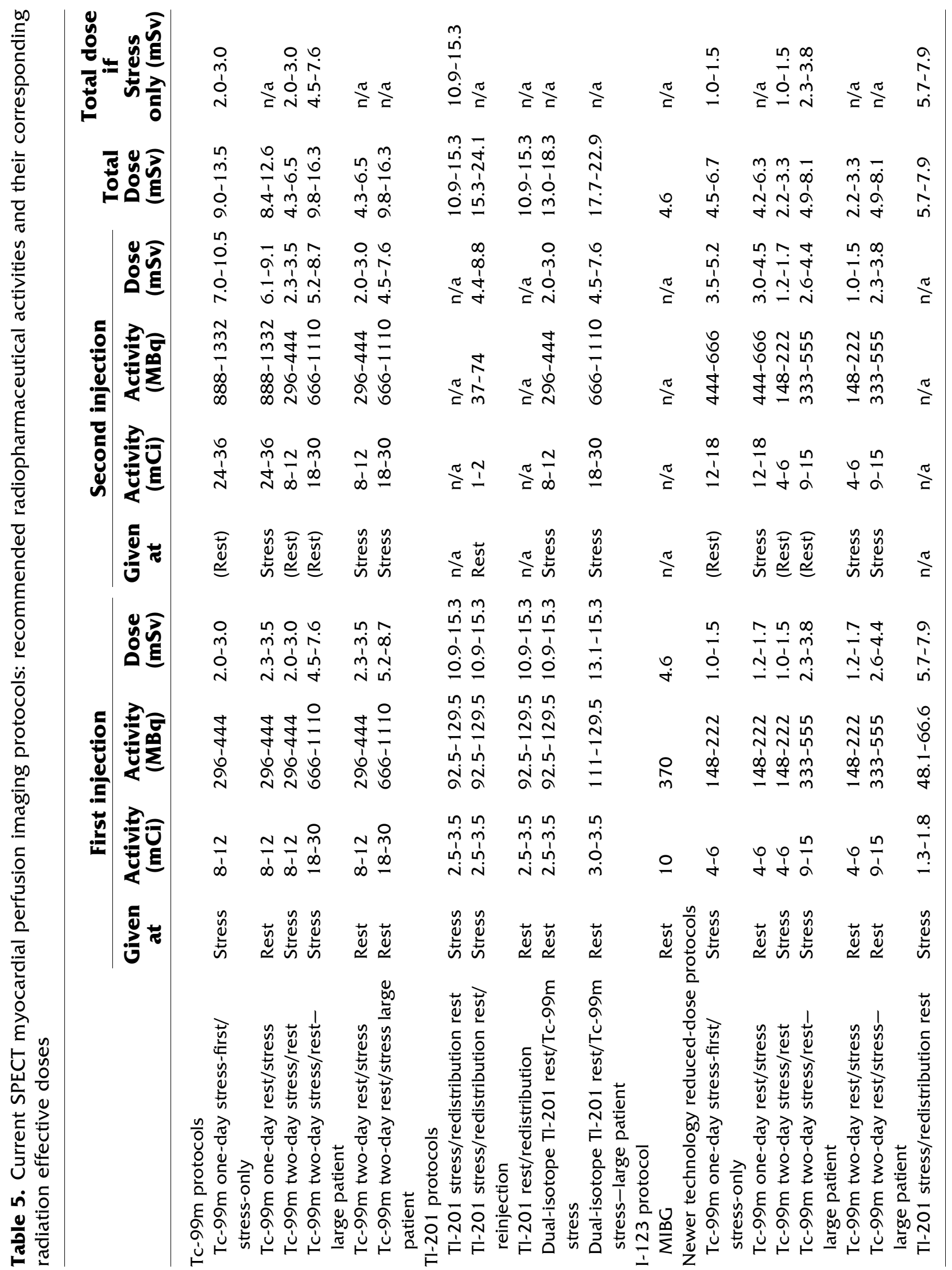




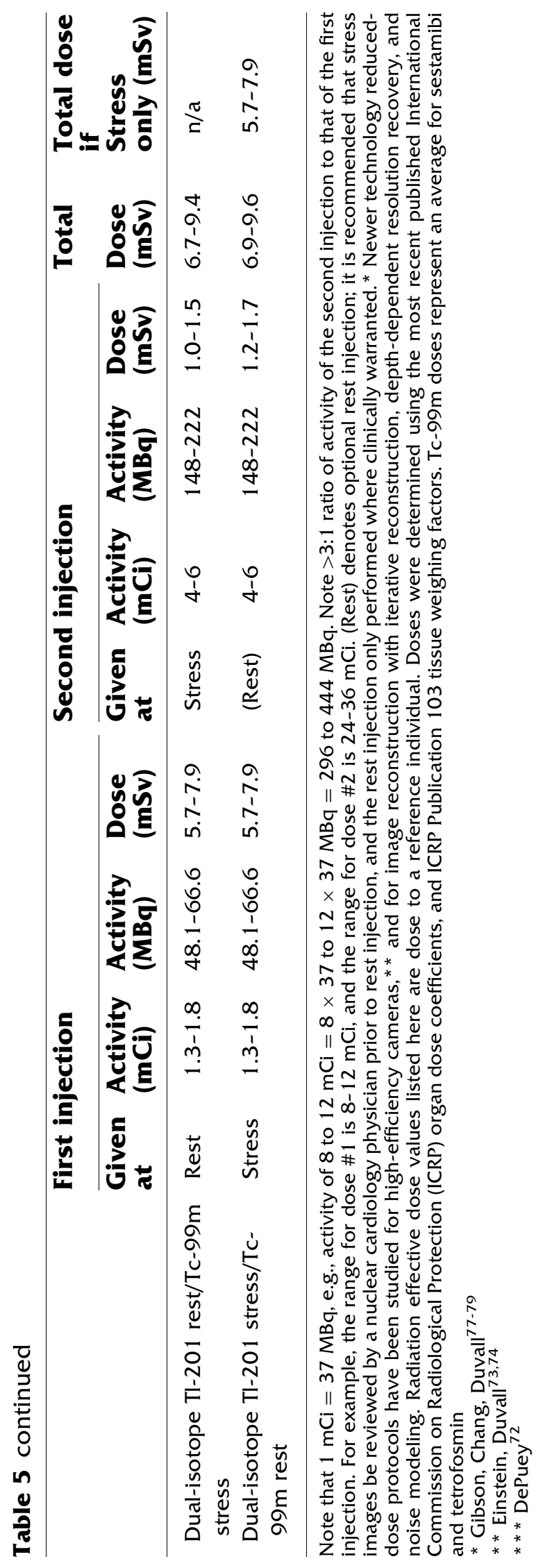

